\section{mAbs from}

\section{mammalian cells}

\section{By Michael J. Haas, Senior Writer}

Cytos Biotechnology AG has developed an antibody platform that employs mammalian cell display to isolate high-affinity human antibodies faster-and possibly with greater diversity-than phage display. ${ }^{1}$ The company already has identified a possible development candidate for nicotine addiction through a single round of screening on a modified version of its cloning platform.

Since publishing those results in the Proceedings of the National Academy of Sciences, the research team-led by senior scientist Roger Beerli-has used the platform to isolate several antibodies against $\mathrm{HBV}$ and the influenza A virus and has those $\mathrm{mAbs}$ in preclinical development.

Beerli told SciBX that the platform yields high-affinity antibodies in a matter of weeks. And because the antibodies are human, they "do not require further optimization, such as humanization or affinity maturation."

Companies polled by $S c i B X$ agreed the platform is a new method for isolating human antibodies but differed about whether its potential advantages over phage display outweighed its apparent limitations.

\section{Matter of magnitude}

In developing the new platform, Beerli's team modified Cytos' DELphi Sindbis system, which already used mammalian cells to express protein libraries, to enable it to clone and display antibodies as well. The DELphi system was originally designed to express RNA in a Sindbis virus vector that transfects hamster cells. Each cell is infected by only one virus, thereby limiting a given cell to the production of one type of protein. After cloning the proteins, the cells are screened and sorted by flow cytometry. ${ }^{2,3}$

For antibody display, the team modified the hamster cells with a transmembrane domain and a surface signaling peptide, thereby allowing antibodies expressed within the cell to display on the cell surface for screening purposes.

Typically, phage display uses all of the B cells from a human donor or donors to generate libraries containing tens of billions of antibodies. Mammalian cell display cannot readily screen a library of that size, so the Cytos team produced a more manageable library of a few million antibodies by selecting only donor B cells specific to a desired antigen.
Beerli did not think that the small size of the antibody libraryorders of magnitude smaller than a typical phage-display library-was a disadvantage. "All of the antigen-specific antibodies are present" by virtue of the preselection of antigen-specific B cells, he said.

Indeed, after isolating nicotine-specific B cells from a participant in a Phase II trial of the company's Nic002 nicotine addiction vaccine, the team generated two antibody libraries on the modified DELphi Sindbis platform. A single round of screening yielded seven human $\mathrm{mAbs}$ with nanomolar affinity for nicotine.

Six of the antibodies significantly inhibited nicotine from crossing the blood-brain barrier in mice. The antibody Nic002 had the highest binding affinity for nicotine $(12 \mathrm{nM})$ and lowered the brain concentration of the substance by a factor of three compared with that seen in control mice.

In 2005, Nic002 (formerly CYT002-NicQb) failed to show a significant increase on the primary endpoint of abstinence in a Swiss Phase II trial in 341 smokers. However, 57\% of a subgroup of patients with a high antibody response to the vaccine achieved continuous abstinence, compared with $32 \%$ for both medium and low responders and $31 \%$ for placebo patients. The company has since improved the compound's potency and in 2007 granted exclusive worldwide rights to Novartis AG. ${ }^{4}$

"Our technology makes full use of human immune system and human affinity maturation machinery to isolate high-affinity antibodies of entirely human origin," said Beerli. "And the screening method primarily identifies highaffinity mAbs that can be produced easily in large-scale quantities."
-Roger Beerli, iotechnology AG
Bassil Dahiyat, president and CEO of Xencor Inc., said the mammalian cell display platform "seems to be an efficient approach with nice molecular biology tools that are very efficient. It is one of several tools that are pushing antibody discovery in a new direction."

But he added: "It isn't tremendously differentiated from existing mammalian display tools" or other methods using B cells from patients who have been exposed to antigen.

For example, Spaltudaq Corp. develops therapeutic antibodies to treat cancer and infectious diseases by isolating B cells from human tumors or human blood and converting them into IgG-secreting cells. $^{5}$

\section{Abs work out}

Besides noting the need for only a single round of screening, Beerli's team wrote in PNAS that its mammalian cell display platform obviates the need for antibody humanization and avoids biases introduced by other screening methods like phage display. However, the use of antigen-specific B cells could limit the platform's utility to a narrow range of indications.

Phage display uses bacteria to express antibodies followed by multiple rounds of screening to isolate antibodies with high affinity 
for the desired antigen. But according to Beerli, the expression and screening processes can be driven by physicochemical properties other than antigen binding - to the detriment of the resulting set of isolated antibodies.

He said phage thus may produce antibodies with the ability to fold properly in the absence of the usual chaperone molecules, to form disulphide bonds in the absence of the usual enzymes or to fold correctly without disulfide bonds at all.

Beerli said these selection biases can result in the isolation of an overabundance of particular clones or antibodies that do not express well for later scale-up and production in mammalian cells.

Additionally, bacteria do not have the same intracellular machinery as mammalian cells. Such differences are thought to limit the types of antibodies that phage display can express. ${ }^{6-8}$ In contrast, Beerli's team wrote in PNAS that expression in mammalian cells "ensures that all of the cellular components normally involved in antibody synthesis and processing are available," resulting in antibodies that sample more widely from the human repertoire than those isolated by phage display.

Thomas Chittenden, executive director of research at ImmunoGen Inc., said Cytos' platform is an elegant system for mammalian cell display and agreed with Beerli that one possible advantage is its ability to isolate human antibodies.

"The value of the technology comes down to how important it is to have fully human antibodies instead of humanized antibodies from mice," thus avoiding the potential for immunogenicity, he said.

But Chittenden did say the question of whether human antibodies have any immunogenic advantages over humanized antibodies is still open to debate. "In the treatment of chronic conditions such as rheumatoid arthritis, fully human antibodies might be preferable," he said. "But if that isn't important-say, for treating an acute infection-then maybe it's not a major advantage."

Added Chittenden: "There are plenty of humanized and even chimeric antibodies" that do not appear to be immunogenic.

ImmunoGen develops antibody-drug conjugates to treat cancer.

Jürgen Hess, manager of scientific affairs at Trion Pharma GmbH, said Cytos' technology "represents a time-saving advance in antibody screening," primarily because it begins with antigen-specific B cells that have already been matured in vivo-thereby obviating the need for further affinity maturation.

Hans de Haard, senior director of discovery research at Ablynx N.V., agreed with Hess, noting that affinity maturation might introduce instability, changed specificity or immunogenicity in the resulting antibodies. "The beauty is the selection of limited numbers of antigen-specific B cells-prior to amplification of antibody genes-that guarantees the creation of good quality and functional libraries," he said.

de Haard also agreed with Beerli that the platform avoids the biases and limitations associated with display systems that do not use mammalian cells. "These limitations fall away, and potentially antibody fragments that can not be produced in E. coli and yeast [displays] can be selected in Cytos' system," he said.
On the other hand, ImmunoGen's Chittenden suggested the time and effort saved by a single round of screening on the Cytos platformversus three or four rounds of panning on phage display-might be offset by the preselection of the B cells from a human donor. "It is an incremental advantage at best," he said. "A round of phage display panning takes a week or two. So yes, the mammalian cell display saves time-but is this a major advantage?"

But Chittenden acknowledged that not having to do postisolation affinity maturation might save significant time compared to the in vitro affinity maturation required for synthetic antibodies isolated by phage display.

\section{Display cases}

Possible advantages over phage display aside, several companies cautioned that the Cytos platform might not be extendable to a wide range of disease targets because of its dependence on antigen-specific B cells.

Said Trion's Hess: “This technology does not represent a straightforward method, as suitable B cell donors have to be identified first." $\mathrm{He}$ suggested this could limit the platform's application to acute or chronic infectious diseases, especially if the B cells had to be isolated from immunized donors.

Ablynx's de Haard agreed this was a drawback because "active immunization of humans with human target proteins cannot be done and is unethical" and thus would prevent application of the platform to inflammation and cancer targets.

"It is not a platform solution for a wide range of antibody targets," said Chittenden.

"It might be best suited for generating antibodies against a subset of antibody targets-such as pathogens of infectious disease or foreign antigens like nicotine-but not against cancer or self-antigens."

Xencor's Dahiyat pointed out that Beerli team's had purposely immunized a human volunteer to generate the necessary antigenspecific B cells-a fact that he thought limited the scope of the proof of concept. "The way they did it maximized their chances of finding good B cells," he said.

Dahiyat said the study would have been more convincing if the team had chosen an antigen of clinical relevance, chosen a patient exposed to it naturally by infection, cancer or autoimmune disease, and then determined whether antibodies could be isolated as efficiently as the antinicotine ones.

Beerli disagreed that his team had stacked the deck by using an immunized donor. He said he expected the platform would work just as well when starting from a donor who had been naturally exposed to antigen as it did when starting from an immunized donor.

In fact, in the PNAS report, Beerli's team disclosed they found an individual with high levels of an antibody against the hormone ghrelin in a group of about 50 donors, though they did not present those results in the paper.

The team also noted that high levels of antibodies against cytokines such as IL- 6 and IL- $1 \alpha$ have been found in humans at reasonably high frequencies, ${ }^{9,10}$ - suggesting that antigen-specific B cells could be isolated from nonimmunized donors. 


\section{DRUG PLATFORMS}

"I would be very much interested in seeing if indeed cytokine-specific human antibodies can be identified with Cytos' system," De Haard said. "It would be a big breakthrough if and when the Cytos team is able to generate fully human antibodies against, for instance, IL-6 from human donors who seem to have a serum response against the cytokine."

Jan van de Winkel, CSO of Genmab A/S agreed. "There are documented cases where inflammation disease patients already have antiIL-6, anti-IL-1, anti-TNF- $\alpha$ [tumor necrosis factor- $\alpha$ ] and anti-IL-12 antibodies," he said. It would have been more interesting to see whether the Cytos platform could isolate antibodies against these targets rather than against viruses or nicotine, he said.

\section{Whence the B cells?}

Looking ahead, Beerli said "isolation of cytokine-specific mAbs is one of many possible avenues we could take from here." But he noted his team is focused on the preclinical development of several mAbs against undisclosed HBV and influenza A antigens.

In isolating those antiviral $\mathrm{mAbs}$ using the new platform, the team began with B cells "from people who were prescreened by ELISA for the presence of antibodies with the right specificity," he said.

Beerli said Cytos eventually might develop Nic002, the most promising antinicotine antibody identified in the PNAS study, but it has no immediate plans to do so.
Cytos has submitted patent applications covering the adaptation of the DELphi Sindbis platform for the display antibodies on mammalian cells and for any antibodies isolated with the modified platform.

\section{REFERENCES}

1. Beerli, R. et al. Proc. Natl. Acad. Sci. USA; published online Sept. 29, 2008; doi:10.1073/pnas.0805942105

Contact: Roger R. Beerli, Cytos Biotechnology AG, Schlieren, Switzerland

e-mail: roger.beerli@cytos.com

2. Koller, D. et al. Nat. Biotechnol. 19, 851-855 (2001)

3. Gatto, D. et al. J. Immunol. 173, 4308-4316 (2004)

4. Maggos, C. BioCentury 15(20), A16; April 30, 2007

5. Mikulski, M. BioCentury 15(18), A14; April 16, 2007

6. Ulrich, H. et al. Proc. Natl. Acad. Sci. USA 92, 11907-11911 (1995)

7. Vaughan, T. et al. Nat. Biotechnol. 14, 309-314 (1996)

8. Bowley, R. et al. Protein Eng. Des. Sel. 20, 81-90 (2007)

9. Galle, P. et al. Eur. J. Immunol. 34, 3267-3275 (2004)

10. Jouvenne, P. et al. Scand. J. Immunol. 46, 413-418 (1997)

\section{COMPANIES AND INSTITUTIONS MENTIONED}

Ablynx N.V. (Euronext:ABLX), Ghent, Belgium Cytos Biotechnology AG (SWX:CYTN), Schlieren, Switzerland Genmab A/S (CSE:GEN), Copenhagen, Denmark ImmunoGen Inc. (NASDAQ:IMGN), Cambridge, Mass. Novartis AG (NYSE:NVS; SWX:NOVN), Basel, Switzerland Spaltudaq Corp., Seattle, Wash.

Trion Pharma GmbH, Munich, Germany Xencor Inc., Monrovia, Calif. 